Supporting Information

\title{
Atomic Dispersion and Surface Enrichment of Palladium in Nitrogen-Doped Porous Carbon Cages Leads to High-Performance Electrocatalytic Reduction of Oxygen
}

\author{
Qiming Liu, ${ }^{a}$ Yi Peng, ${ }^{a}$ Qiaoxia Li,${ }^{a, b}$ Ting He,${ }^{a, c}$ David Morris, ${ }^{d}$ Forrest Nichols, ${ }^{a}$ Rene \\ Mercado, ${ }^{a}$ Peng Zhang, ${ }^{d}$ Shaowei Chen ${ }^{a, *}$
}

${ }^{a}$ Department of Chemistry and Biochemistry, University of California, 1156 High Street, Santa Cruz, California 95064, United States

${ }^{\mathrm{b}}$ Shanghai Key Laboratory of Materials Protection and Advanced Materials in Electric Power, College of Environmental and Chemical Engineering, Shanghai University of Electric Power, 2588 Changyang Road, Yangpu District, Shanghai 200090, China

${ }^{c}$ Hunan Provincial Key Laboratory of Chemical Power Sources, College of Chemistry and Chemical Engineering, Central South University, Changsha, Hunan 410083, China

${ }^{\mathrm{d}}$ Department of Chemistry, Dalhousie University, 6274 Coburg Road, Halifax, NS B3H 4R2, Canada

*E-mail: shaowei@ucsc.edu

\section{List of Contents}

- 12 figures

- 5 tables 


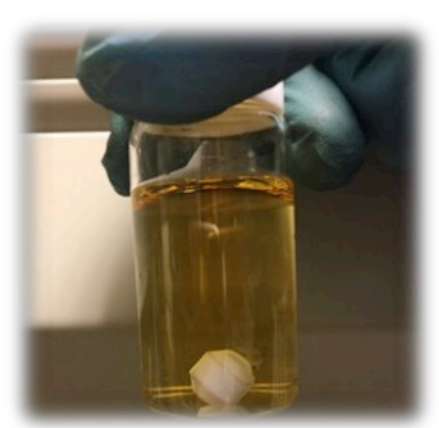

Before

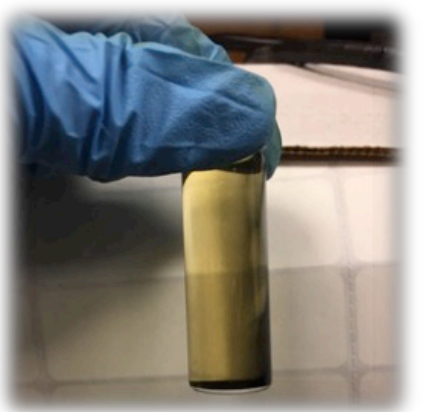

After

Figure S1. Pd-HNC ${ }_{10}$ before and after thermal refluxing. The resulting supernatant demonstrates a similar yellowish color, indicating that the HNC is saturated with Pd centers.

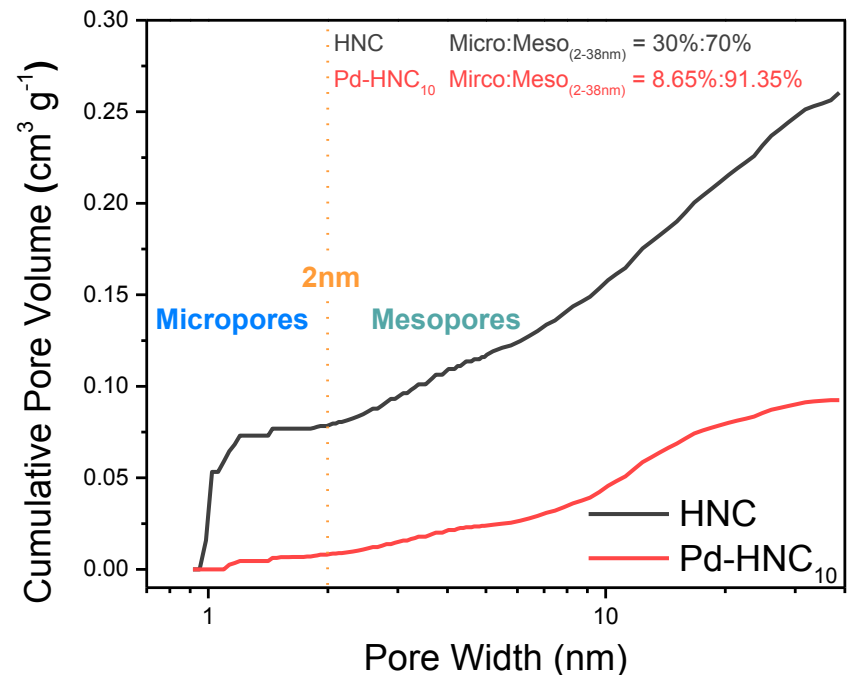

Figure S2. Cumulative pore volume as a function of pore width of the $\mathrm{HNC}$ and $\mathrm{Pd}-\mathrm{HNC}_{10}$ samples. 


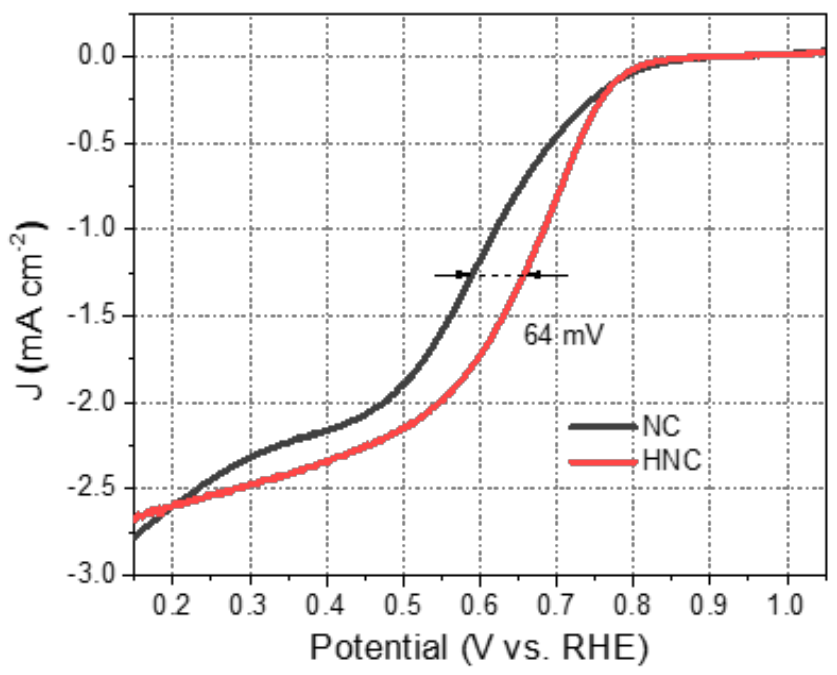

Figure S3. Comparison of the ORR performance of HNC and solid N-doped carbon (NC) at the rotation rate of $1600 \mathrm{rpm}$ in oxygen-saturated $0.1 \mathrm{M} \mathrm{KOH}$.

Following the same procedure but without the etching process, we directly pyrolyzed ZIF-8 precursors into solid N-doped carbon (NC). The corresponding ORR polarization curves are shown in Figure S3. One can see that HNC clearly outperformed the NC sample, with an $\mathrm{E}_{1 / 2}$ that is $64 \mathrm{mV}$ more positive. Meanwhile, the diffusion-limited current of HNC was also larger than that of NC, suggesting that the hollow structure is indeed conducive to the ORR electrocatalysis.
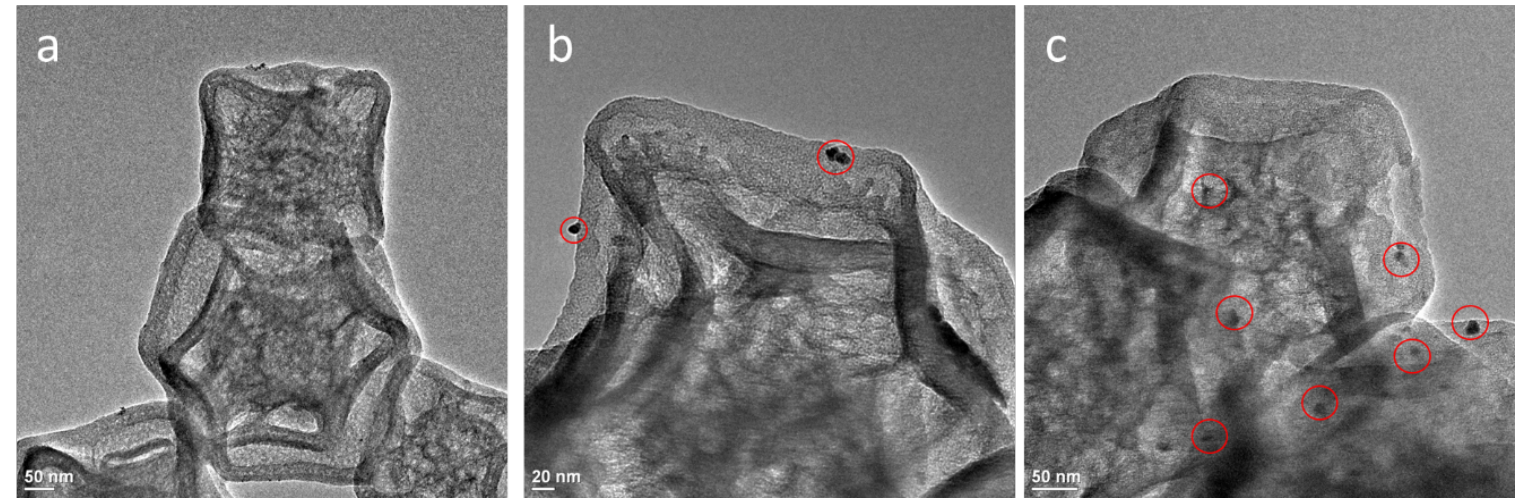

Figure S4. TEM images of (a) Pd-HNC highlight the few palladium nanoparticles. 

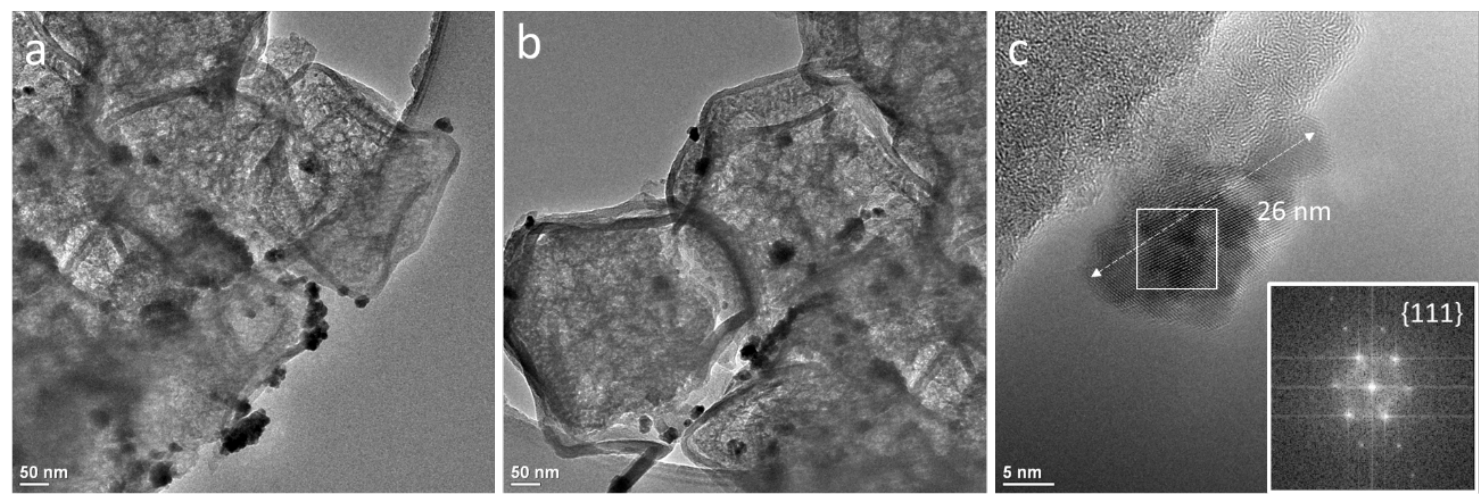

Figure S5. (a-c) TEM images of PdNP/HNC at varied magnifications. Inset to panel (c) is the corresponding Fast Fourier Transform (FFT) pattern of the Pd nanoparticles in the white square.
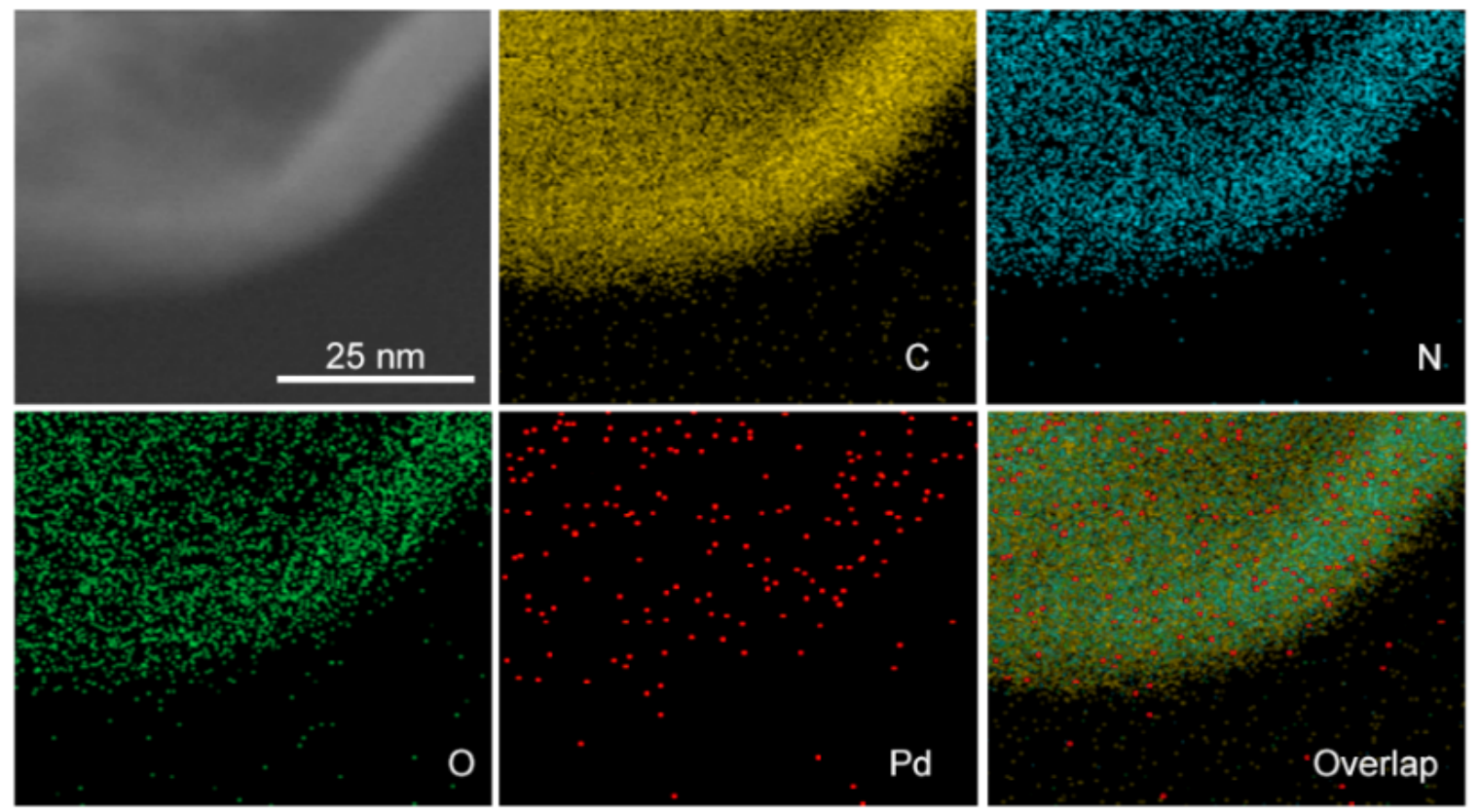

Figure S6. HAADF-STEM images of the edge side (without apparent particles) of Pd-HNC ${ }_{10}$ and the corresponding elemental maps of carbon, nitrogen, oxygen, palladium, and overlap. 


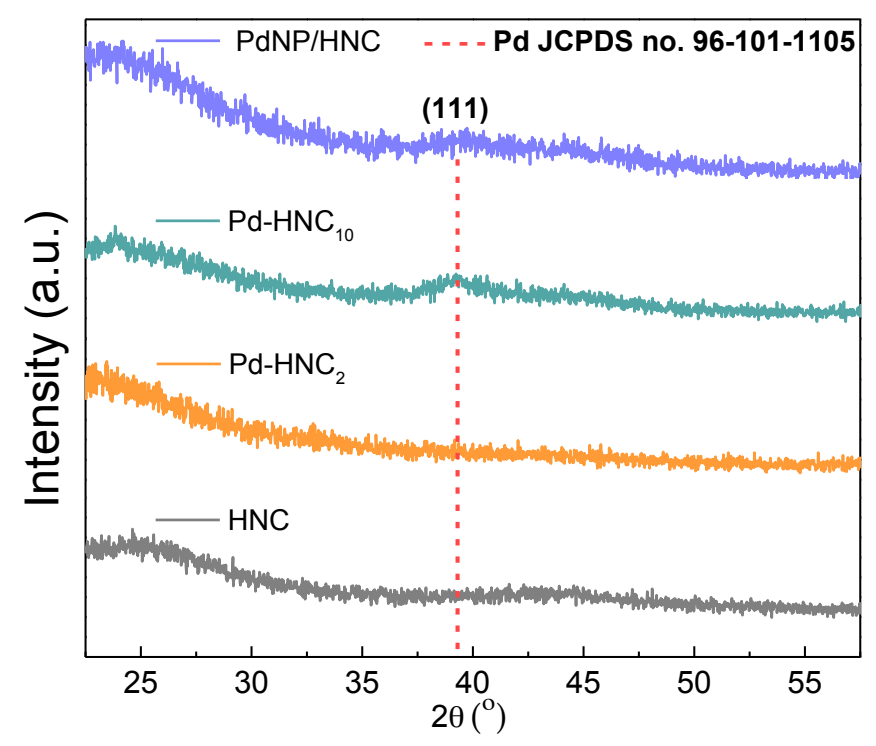

Figure S7. XRD patterns of $\mathrm{PdNP} / \mathrm{HNC}, \mathrm{Pd}-\mathrm{HNC}_{10}, \mathrm{Pd}-\mathrm{HNC}_{2}$, and $\mathrm{HNC}$. Dashed lines indicate the expected $2 \theta$ position of the (111) diffractions of $f c c$ Pd (JCPDS no. 96-101-1105). Note that Pd-HNC ${ }_{10}$ contained more metallic palladium than PdNP/HNC based on results from XPS measurements.

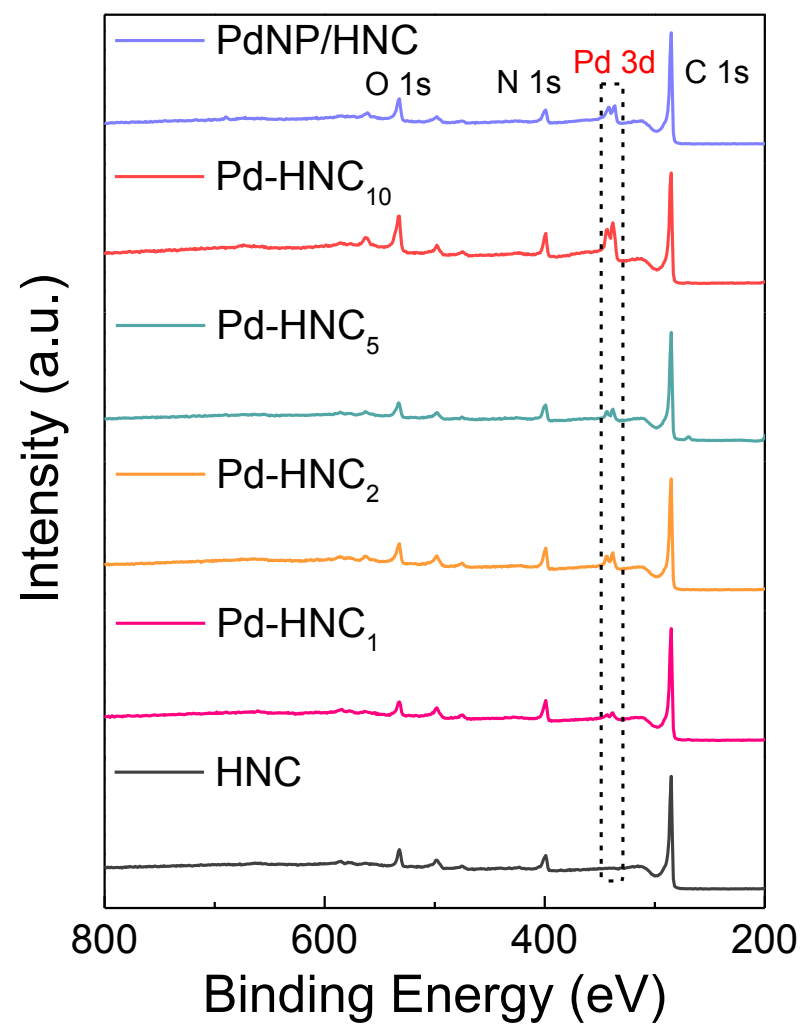

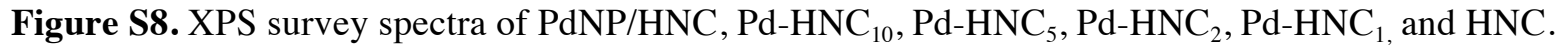



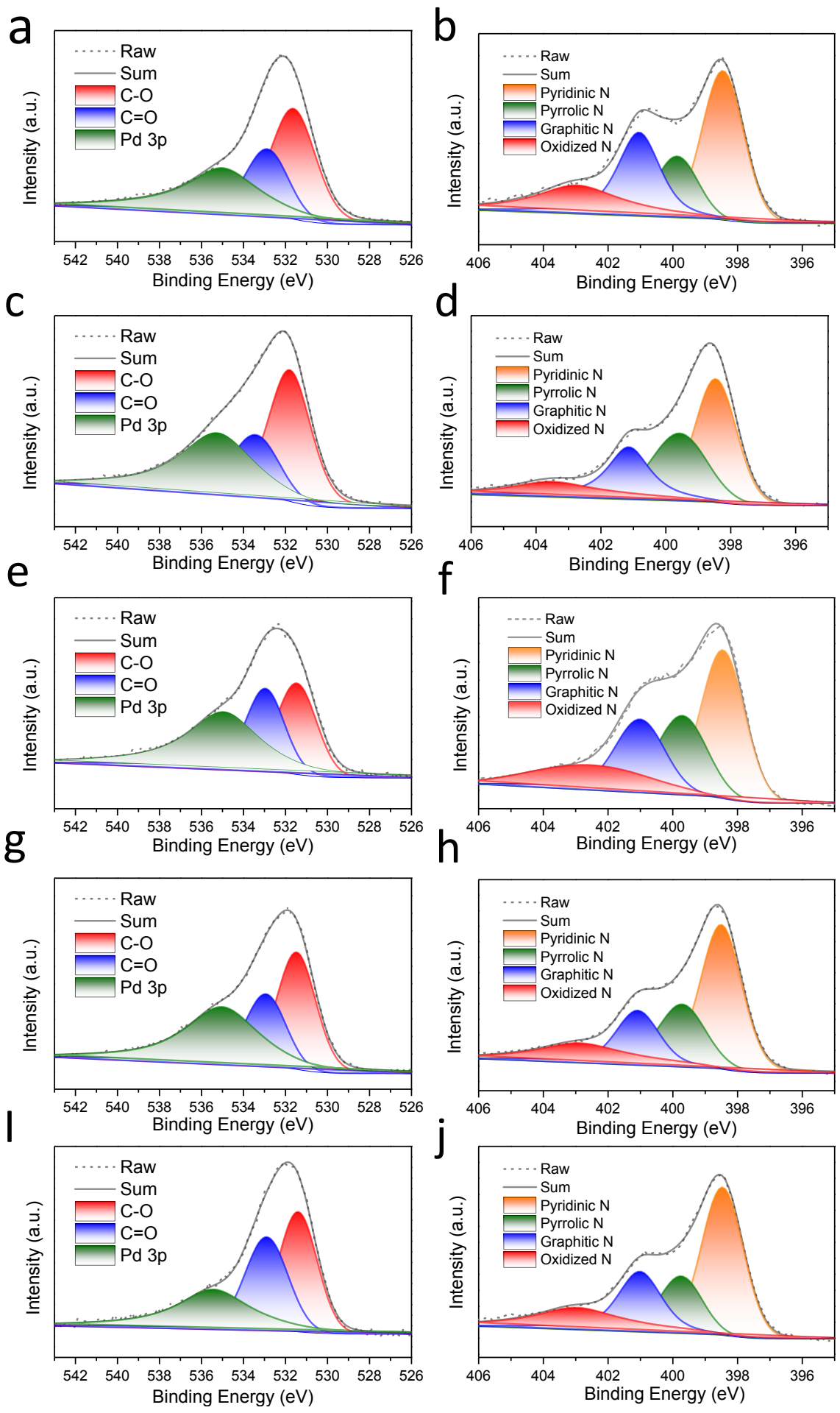

Figure S9. High-resolution XPS spectra of the (left panels) $\mathrm{O} 1 \mathrm{~s}$ and (right panels) $\mathrm{N} 1 \mathrm{~s}$ electrons of $(\mathrm{a}, \mathrm{b})$ PdNP/HNC, (c, d) Pd-HNC 10 , (e, f) Pd-HNC 5 , (g, h) Pd-HNC 2 , and (i, j) Pd-HNC P $^{\text {Pd }}$ 

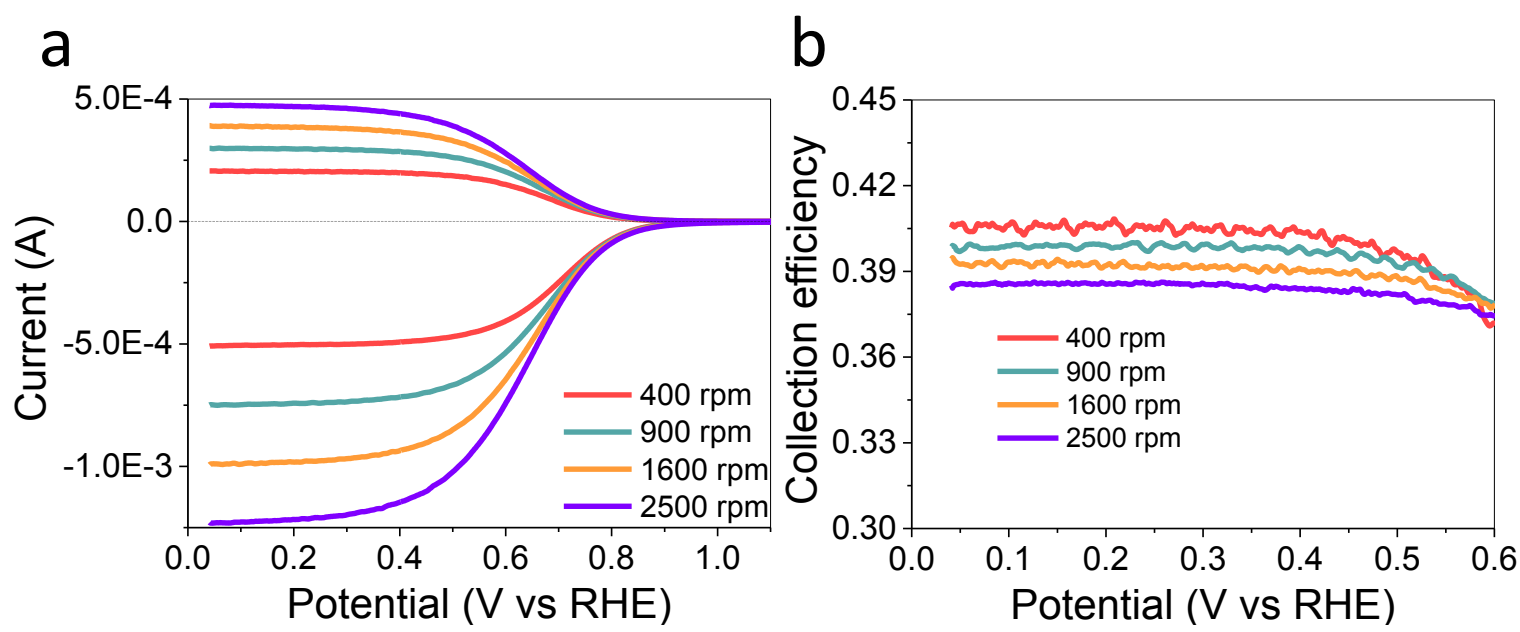

Figure S10. (a) Collection experiments by RRDE voltammetry at the rotation rates of 400 to $2500 \mathrm{rpm}$ in $0.1 \mathrm{M} \mathrm{KOH}$ with $10 \mathrm{mM} \mathrm{K}_{3} \mathrm{Fe}(\mathrm{CN})_{6}$. The ring electrode potentia is set at $+0.2 \mathrm{~V}$ (b) Collection efficiency $(\mathrm{N})$ calculated from the ring and disk currents at varied electrode potentials.

The procedure to determine the collection efficiency $(\mathrm{N})$ of the ring electrode was adopted from ref. 1. The electrolyte was $0.1 \mathrm{M} \mathrm{KOH}$ with $10 \mathrm{mM} \mathrm{K}_{3} \mathrm{Fe}(\mathrm{CN})_{6}$. RRDE voltammograms was acquired at the rotation rates of 400 to $2500 \mathrm{rpm}$ (Figure S10a). On the disk electrode, ferricyanide was reduced into ferrocyanide, $\mathrm{Fe}(\mathrm{CN})_{6}^{3-}+e^{-} \rightarrow \mathrm{Fe}(\mathrm{CN})_{6}^{4-}$, part of which diffused to the ring electrode and was oxidized back into ferricyanide, $\mathrm{Fe}(\mathrm{CN})_{6}^{4-} \rightarrow \mathrm{Fe}(\mathrm{CN})_{6}^{3-}+e^{-}$. The disk and ring limiting currents were then used to calculate the empirical collection efficiency (N, Figure $\mathrm{S} 10 \mathrm{~b}$ ), $N=\left|\frac{i_{\text {Ring }}}{i_{\text {Disk }}} \frac{n_{D}}{n_{R}}\right|$, where $\mathrm{n}_{\mathrm{D}}$ and $\mathrm{n}_{\mathrm{R}}$ are the numbers of electron transfer of the disk and ring reactions. From Figure S10b, the average collection efficiency $(\mathrm{N})$ is estimated to be 0.40 . 


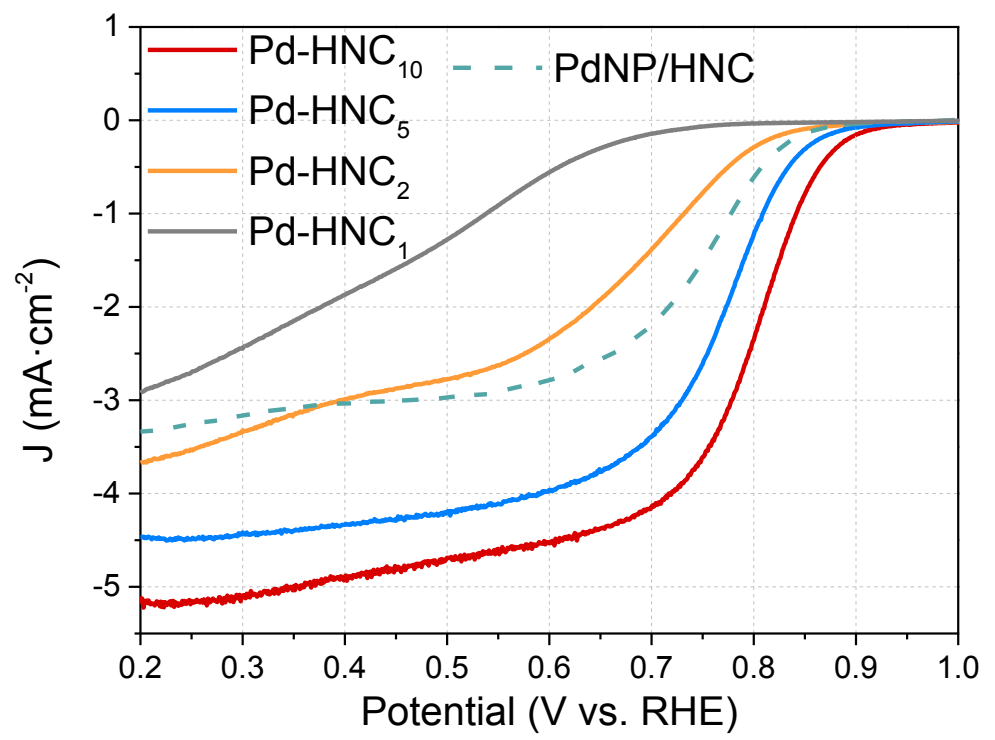

Figure S11. Linear sweep voltammograms (LSVs) of Pd-HNC nanocomposites and PdNP/HNC at the rotation rate of $1600 \mathrm{rpm}$ in $0.1 \mathrm{M} \mathrm{HClO}_{4}$.

It should be noted that in acid, $\mathrm{Pd}-\mathrm{HNC}_{2}$ exhibits an ORR activity worse than PdNP/HNC, possibly because of the lack of neighboring metal atoms around the atomically dispersed $\mathrm{Pd}$ species that are crucial to acidic ORR. ${ }^{2}$ In fact, the $\mathrm{Pd}-\mathrm{HNC}_{1}$ sample performs even worse in acid than in base.

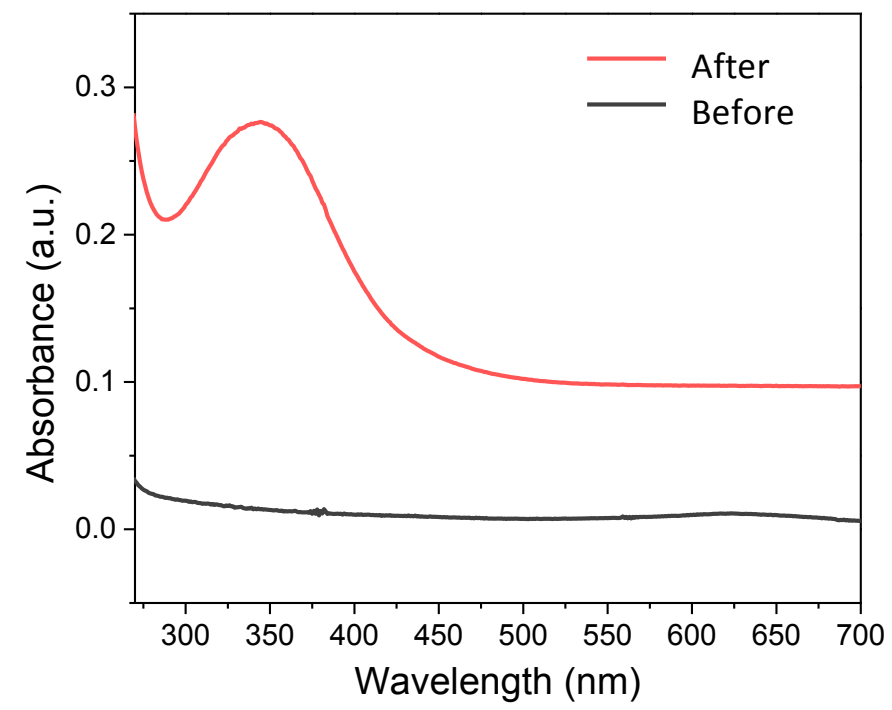

Figure S12. UV-Vis spectra of the supernatant solution collected from the $\mathrm{Pd}-\mathrm{HNC}_{10}$ sample before and after EDTA treatment. 
Table S1. Pd contents in the series of samples as determined by ICP-OES and XPS measurements.

\begin{tabular}{|c|c|c|c|c|c|}
\hline Samples & $\mathrm{Pd}-\mathrm{HNC}_{1}$ & $\mathrm{Pd}-\mathrm{HNC}_{2}$ & Pd-HNC ${ }_{5}$ & Pd-HNC 10 & $\mathrm{PdNP} / \mathrm{HNC}$ \\
\hline $\begin{array}{c}\mathrm{Pd}(\mathrm{wt} \%) \\
\text { calculated from ICP- } \\
\text { OES }\end{array}$ & 0.87 & 1.75 & 4.36 & 7.77 & 2.61 \\
\hline $\begin{array}{c}\text { Pd(II) (wt } \%) \\
\text { calculated from XPS }\end{array}$ & 0.74 & 1.43 & 3.39 & 5.42 & 1.14 \\
\hline
\end{tabular}

Table S2. Pd 3d binding energies and concentrations in the series of sample as determined by XPS measurements.

\begin{tabular}{|c|c|c|c|c|c|c|c|c|}
\hline Samples & \multicolumn{2}{|c|}{$\mathrm{Pd}^{0}(\mathrm{eV})$} & \multicolumn{2}{|c|}{$\mathrm{Pd}^{2+}(\mathrm{eV})$} & $\mathrm{Pd}^{2+}: \mathrm{Pd}^{0}$ & $\mathrm{Pd}$ at\% & $\mathrm{Pd} \mathrm{wt \%}$ & $\mathrm{Pd}: \mathrm{N}$ \\
\hline $\mathrm{PdNP} / \mathrm{HNC}$ & 335.805 & 341.065 & 337.736 & 342.996 & 0.77 & 1.27 & 9.88 & $1: 6.7$ \\
\hline $\mathrm{Pd}-\mathrm{HNC}_{10}$ & 335.74 & 341.00 & 337.897 & 343.157 & 2.3 & 2.45 & 17.48 & $1: 4.6$ \\
\hline $\mathrm{Pd}_{-} \mathrm{HNC}_{5}$ & 335.875 & 341.135 & 337.961 & 343.221 & 3.5 & 0.81 & 6.58 & $1: 11.2$ \\
\hline $\mathrm{Pd}_{-} \mathrm{HNC}_{2}$ & 335.913 & 341.173 & 338.014 & 343.274 & 4.5 & 0.97 & 7.74 & $1: 11.6$ \\
\hline $\mathrm{Pd}-\mathrm{HNC}_{1}$ & 335.595 & 340.855 & 338.040 & 343.300 & 5.6 & 0.43 & 3.55 & $1: 26.1$ \\
\hline
\end{tabular}

Table S3. Comparison of the content of atomic Pd species between Pd-HNC $\mathrm{H}_{10}$ and relevant Pd SACs in the literature.

\begin{tabular}{|c|c|c|}
\hline Samples & Atomic Pd content (wt $\%)$ & Reference \\
\hline $\mathrm{Pd}-\mathrm{HNC}_{10}$ & 5.42 & This work \\
\hline $\mathrm{Pd}-\mathrm{SAs}$ & 0.16 & 3 \\
\hline $\mathrm{Pd}_{1} / \mathrm{C}_{3} \mathrm{~N}_{4}$ & 0.5 & 4 \\
\hline $\mathrm{Pd} / \mathrm{N}-\mathrm{CNTs}$ & 0.5 & 5 \\
\hline$[\mathrm{Pd}] \mathrm{mpg}-\mathrm{C}_{3} \mathrm{~N}_{4}$ & 0.5 & 6 \\
\hline $\mathrm{RGO} @ \mathrm{AC} / \mathrm{Pd} \mathrm{SACs}$ & 0.4 & 7 \\
\hline $\mathrm{Pd} 1 \% \mathrm{NCNT}$ & 0.9 & 8 \\
\hline
\end{tabular}

Table S4. Summary of the fitting results of the EXAFS spectra of $\mathrm{Pd}-\mathrm{HNC}_{10}, \mathrm{Pd}-\mathrm{HNC}_{2}$, and $\mathrm{Pd}$ foil.

\begin{tabular}{|c|c|c|c|c|c|c|}
\hline Samples & bond & $\mathrm{CN}$ & $\mathrm{R}(\AA)$ & $\sigma^{2}(\AA) \times 10^{-3}$ & $\mathrm{E}_{0}(\mathrm{eV})$ & $\mathrm{R}$ factor \\
\hline Pd Foil & Pd-Pd & 12 (Fixed) & $2.741(2)$ & $5.2(3)$ & $1.4(4)$ & \multirow{2}{*}{0.0048} \\
\hline \multirow{2}{*}{ Pd-HNC $_{10}$} & $\mathrm{Pd}-\mathrm{Pd}$ & $2.2(4)$ & $2.797(6)$ & $7(1)$ & $2.9(7)$ & \multirow{2}{*}{0.0029} \\
\cline { 2 - 6 } & $\mathrm{Pd}-\mathrm{N}$ & $3.4(3)$ & $2.004(5)$ & $4(1)$ & $2.9(7)$ & \\
\hline \multirow{2}{*}{$\mathrm{Pd}^{-\mathrm{HNC}_{2}}$} & $\mathrm{Pd}-\mathrm{Pd}$ & $2.1(8)$ & $2.80(2)$ & $7(3)$ & $6(2)$ & \multirow{2}{*}{0.0213} \\
\cline { 2 - 6 } & $\mathrm{Pd}-\mathrm{N}$ & $5(1)$ & $2.07(2)$ & $10(3)$ & $6(2)$ & \\
\hline
\end{tabular}

Note: $\mathrm{CN}$, coordination number; $\mathrm{R}$, interatomic distance; $\sigma^{2}$, Debye-Waller factor; and $\mathrm{E}_{0}$, edge energy shift. The $\mathrm{R}$ factor is used to value the goodness of the fitting. 
Table S5. Comparison of the ORR activity between Pd-HNC and relevant Pd-based catalysts in the literature in $0.1 \mathrm{M} \mathrm{KOH}$

\begin{tabular}{|c|c|c|c|c|c|}
\hline Samples & $\begin{array}{c}\text { Catalyst }_{\text {metal }} \\
\text { Loading }\left(\mu \mathrm{g} \mathrm{cm}^{-2}\right)\end{array}$ & $\begin{array}{c}\mathrm{E}_{1 / 2} \\
(\mathrm{~V} \text { vs. RHE) }\end{array}$ & $\mathrm{n}$ & $\mathrm{J}_{\mathrm{k}}(\mathrm{A} / \mathrm{mg})$ & $\begin{array}{l}\text { References } \\
\text { (Year) }\end{array}$ \\
\hline Pd-HNC 10 & 12.58 & 0.898 & 3.97 & $0.30 @+0.85 \mathrm{~V}$ & This Work \\
\hline Pd-HNC 5 & 7.09 & 0.889 & 3.95 & $0.49 @+0.85 \mathrm{~V}$ & This Work \\
\hline Pd-HNC ${ }_{2}$ & 2.84 & 0.846 & 3.94 & $0.65 @+0.85 \mathrm{~V}$ & This Work \\
\hline Au-O-PdZn & 40 & $\sim 0.9$ & 3.96 & $0.105 @+0.90 \mathrm{~V}$ & $9(2019)$ \\
\hline Pd HBNDs & 50 & 0.863 & $\sim 4$ & 1 & $10(2019)$ \\
\hline $\begin{array}{c}\text { fct- } \\
\text { PdFe@Pd@NG }\end{array}$ & / & 0.83 & $\sim 3.9$ & l & $11(2018)$ \\
\hline $\mathrm{Pd} / \mathrm{TiO}_{2}-\mathrm{V}_{\mathrm{o}}$ & $\sim 15.12$ & $\sim 0.82$ & 3.8 & 1 & $12(2018)$ \\
\hline $\mathrm{Au} @ \mathrm{Pd}_{0.5}$ & 10.2 & $\sim 0.9$ & 3.8 & $0.5 @+0.85 \mathrm{~V}$ & $13(2018)$ \\
\hline $\begin{array}{c}\text { Pd nanocubes } \\
@ \mathrm{Mo} / \mathrm{C} \\
\end{array}$ & 20.4 & 0.837 & $\sim 3.9$ & $0.11 @+0.85 \mathrm{~V}$ & $14(2018)$ \\
\hline $\begin{array}{c}\mathrm{Pd} / 3 \mathrm{D}-\mathrm{GNS}- \\
\mathrm{L} 90\end{array}$ & $\sim 66$ & $\sim 0.8$ & $\sim 3.9$ & / & $15(2018)$ \\
\hline Pd-Net & 16.9 & 0.84 & 3.98 & $0.62 @+0.85 \mathrm{~V}$ & $16(2018)$ \\
\hline PdNPs & 22.6 & 0.78 & 3.45 & $0.15 @+0.85 \mathrm{~V}$ & $16(2018)$ \\
\hline Pd-N/3D-GNS & $\sim 40$ & $\sim 0.82$ & $\sim 4.0$ & 1 & $17(2017)$ \\
\hline
\end{tabular}




\section{References}

(1) Paulus, U. A.; Schmidt, T. J.; Gasteiger, H. A.; Behm, R. J. Oxygen Reduction on a High-Surface Area Pt/Vulcan Carbon Catalyst: A Thin-Film Rotating Ring-Disk Electrode Study. J. Electroanal. Chem. 2001, 495, 134-145.

(2) Dong, J. C.; Zhang, X. G.; Briega-Martos, V.; Jin, X.; Yang, J.; Chen, S.; Yang, Z. L.; Wu, D. Y.; Feliu, J. M.; Williams, C. T.; Tian, Z. Q.; Li, J. F. In Situ Raman Spectroscopic Evidence for Oxygen Reduction Reaction Intermediates at Platinum Single-Crystal Surfaces. Nat. Energy 2019, 4, 60-67.

(3) Wei, S. J.; Li, A.; Liu, J. C.; Li, Z.; Chen, W. X.; Gong, Y.; Zhang, Q. H.; Cheong, W. C.; Wang, Y.; Zheng, L. R.; Xiao, H.; Chen, C.; Wang, D. S.; Peng, Q.; Gu, L.; Han, X. D.; Li, J.; Li, Y. D. Direct Observation of Noble Metal Nanoparticles Transforming to Thermally Stable Single Atoms. Nat. Nanotechnol. 2018, $13,856-861$.

(4) Huang, X. H.; Xia, Y. J.; Cao, Y. J.; Zheng, X. S.; Pan, H. B.; Zhu, J. F.; Ma, C.; Wang, H. W.; Li, J. J.; You, R.; Wei, S. Q.; Huang, W. X.; Lu, J. L. Enhancing Both Selectivity and Coking-Resistance of a SingleAtom Pd-1/C3n4 Catalyst for Acetylene Hydrogenation. Nano Res. 2017, 10, 1302-1312.

(5) Podyacheva, O. Y.; Bulushev, D. A.; Suboch, A. N.; Svintsitskiy, D. A.; Lisitsyn, A. S.; Modin, E.; Chuvilin, A.; Gerasimov, E. Y.; Sobolev, V. I.; Parmon, V. N. Highly Stable Single-Atom Catalyst with lonic Pd Active Sites Supported on N-Doped Carbon Nanotubes for Formic Acid Decomposition. ChemSusChem 2018, 11, 3724-3727.

(6) Vile, G.; Albani, D.; Nachtegaal, M.; Chen, Z. P.; Dontsova, D.; Antonietti, M.; Lopez, N.; Perez-Ramirez, J. A Stable Single-Site Palladium Catalyst for Hydrogenations. Angew. Chem. Int. Ed. 2015, 54, 11265-11269.

(7) Xi, J. B.; Sung, H. Y.; Wang, D.; Zhang, Z. Y.; Duan, X. M.; Xiao, J. W.; Xiao, F.; Liu, L. M.; Wang, S. Confined-Interface-Directed Synthesis of Palladium Single-Atom Catalysts on Graphene/Amorphous Carbon. Appl. Catal. B: Environ. 2018, 225, 291-297.

(8) Arrigo, R.; Schuster, M. E.; Xie, Z. L.; Yi, Y. M.; Wowsnick, G.; Sun, L. L.; Hermann, K. E.; Friedrich, M.; Kast, P.; Havecker, M.; Knop-Gericke, A.; Schlogl, R. Nature of the N-Pd Interaction in NitrogenDoped Carbon Nanotube Catalysts. ACS Catal. 2015, 5, 2740-2753.

(9) Yang, Y.; Xiao, W. P.; Feng, X. R.; Xiong, Y.; Gong, M. X.; Shen, T.; Lu, Y.; Abruna, H. D.; Wang, D. L. Golden Palladium Zinc Ordered Intermetallics as Oxygen Reduction Electrocatalysts. ACS Nano 2019, 13, 5968-5974.

(10) Fan, C.; Huang, Z. H.; Wang, C.; Hu, X. Y.; Qiu, X. Y.; She, P. L.; Sun, D. M.; Tang, Y. W. HighlyBranched Palladium Nanodandelions: Simple, Fast, and Green Fabrication with Superior Oxygen Reduction Property. Chem. Eur. J. 2019, 25, 4920-4926.

(11) Maiti, K.; Balamurugan, J.; Peera, S. G.; Kim, N. H.; Lee, J. H. Highly Active and Durable Core-Shell Fct-Pdfe@Pd Nanoparticles Encapsulated Ng as an Efficient Catalyst for Oxygen Reduction Reaction. ACS Appl. Mater. Interfaces 2018, 10, 18734-18745.

(12) Li, J.; Zhou, H.; Zhuo, H.; Wei, Z. Z.; Zhuang, G. L.; Zhong, X.; Deng, S. W.; Li, X. N.; Wang, J. G. Oxygen Vacancies on Tio2 Promoted the Activity and Stability of Supported Pd Nanoparticles for the Oxygen Reduction Reaction. J. Mater. Chem. A 2018, 6, 2264-2272.

(13) Xue, Q.; Bai, J.; Han, C. C.; Chen, P.; Jiang, J. X.; Chen, Y. Au Nanowires@Pd-Polyethylenimine Nanohybrids as Highly Active and Methanol-Tolerant Electrocatalysts toward Oxygen Reduction Reaction in Alkaline Media. ACS Catal. 2018, 8, 11287-11295.

(14) Yan, W.; Wu, W.; Wang, K.; Tang, Z. H.; Chen, S. W. Oxygen Reduction Reaction and Hydrogen Evolution Reaction Catalyzed by Carbon-Supported Molybdenum-Coated Palladium Nanocubes. Int. J. Hydrogen Energy 2018, 43, 17132-17141. 
(15) Kabir, S.; Serov, A.; Atanassov, P. 3d-Graphene Supports for Palladium Nanoparticles: Effect of Micro/Macropores on Oxygen Electroreduction in Anion Exchange Membrane Fuel Cells. J. Power Sources 2018, 375, 255-264.

(16) Begum, H.; Ahmed, M. S.; Cho, S.; Jeon, S. Freestanding Palladium Nanonetworks Electrocatalyst for Oxygen Reduction Reaction in Fuel Cells. Int. J. Hydrogen Energy 2018, 43, 229-238.

(17) Kabir, S.; Serov, A.; Artyushkova, K.; Atanassov, P. Nitrogen-Doped Three-Dimensional GrapheneSupported Palladium Nanocomposites: High-Performance Cathode Catalysts for Oxygen Reduction Reactions. ACS Catal. 2017, 7, 6609-6618. 